\title{
Alternative methodology to consider damage and expansions in external sulfate attack modeling
}

Tai Ikumi ${ }^{\text {a, }}$, Sergio H.P. Cavalaro ${ }^{\text {a }}$, Ignacio Segura ${ }^{a}$ and Antonio Aguado ${ }^{a}$

\author{
${ }^{a}$ Department of Construction Engineering, Universitat Politècnica de Catalunya \\ Barcelona Tech, Jordi Girona 1-3, C1, E-08034 Barcelona, Spain
}

* Corresponding author: Tai Ikumi Montserrat. Department of Construction Engineering, Universitat Politècnica de Catalunya Barcelona Tech, Jordi Girona 1-3, C1, E-08034 Barcelona, Spain. Email address: tai.ikumi@estudiant.upc.edu, Tel: +34934016507 Fax: +34934011036

\begin{abstract}
A diffusion - reaction numerical model is proposed to simulate the response of concrete exposed to external sulfate attack. Diffusion properties are modified based on the strain reached and the ratio of porosity filled by ettringite. A direct and intuitive approach is proposed for the consideration of the diffusion in a cracked porous media based on the constitutive law of the material. A methodology to compute expansions based on a more realistic consideration of the concrete porosimetry is presented, by which it is possible to distinguish different strain contributions from different pore sizes. The described approach also allows the consideration of different capacities to accommodate expansive product for each pore size considered and the faster filling rate existent in small pores. Critical parameters of the numerical model developed are recognized and
\end{abstract}


established. Expansions obtained by the new model are in good agreement with experimental data published in the literature.

Keywords: Sulfate Attack (C), Concrete (E), Durability (C), Modeling (E), Pore Size Distribution (B)

\section{1- INTRODUCTION}

Durability of concrete has become a major issue in structural engineering over the last decades. In 2012, it was estimated that $51.2 \%$ of the annual investment in the construction field in EU27 countries (excluding Cyprus, Greece, Luxembourg and Malta) plus Norway and Switzerland, was dedicated to Renovation and Maintenance $(\mathrm{R} \& \mathrm{M})$, turning concrete durability into a key aspect in the industry [1]. Within this framework, restoring and management systems of existing structures gain prominence, where numerical modelization of degradation phenomena plays an essential role. It is important to highlight that in many cases, once a concrete deterioration process is detected in built structures, the only possible action is of the palliative nature, through a monitoring of the structural behavior. For this, the development of prediction models about the future evolution become crucial.

Degradation of concrete exposed to sulfate solutions has been of concern since the early years of the 19th century [2]. The importance of the external sulfate attack in the concrete degradation processes is usually related to the need of assessing the durability of underground nuclear waste containments, tunnel linings and dams, which may be sometimes in contact with sulfate-rich soils [3-6]. The relative significance of this 
phenomenon has been questioned over the last decade and, according to Neville [7], the opinion that sulfate attack is not a widespread problem in concrete structures is expressed even by those who have published extensively on the topic. The fact that there are few reported cases of structures in service damaged by sulfates in the soil or in groundwater is supported by several authors $[7,8]$. Despite all these opinions, most of them based on indisputable facts, now the rate of published papers dealing with the sulfate attack is greater than ever, reflecting a growing interest in the scientific community. This growing interest may be explained by the considerable controversy still existing on basic topics within the sulfate attack phenomenon and the deterioration mechanisms involved, such as the expansion mechanism and the gypsum role on the expansion process [7]. On the other hand, despite structural failures due to sulfate attack are rare, in structures that requires high stability due to a small displacement tolerance, the expansive nature of the sulfate attack should be carefully assessed.

Traditionally, due to the high level of complexity of the external sulfate attack, where transport, chemical and physical processes between sulfate ions and the hydrated compounds of the cement paste are involved $[9,10]$, unilateral efforts have been made into different specific fields to model a specific phenomenon within the overall problem. However, during the last years, a number of advanced comprehensive models have been developed $[3,4,11,12]$, even considering a full tenso-deformational mechanical analysis through a mesostructural representation of the material, able to simulate not only crack formation and propagation, but also its influence on the diffusion-driven process [4]. 
Every possible mechanical framework that may be developed is based on the expansions obtained through the numerical model. Most of the models referred above $[3,4,11]$ obtain the expansions using a simplified expression described by Tixier \& Mobasher [11]. In this expression, the pores capacity to accommodate ettringite is represented by a single buffer coefficient $(f)$. This coefficient is linearly applied to the overall ettringite formation and the overall porosity is considered without differentiating the size of the pores. By this consideration, all the buffer capacity of the matrix has to be filled before the expansions start to be computed. In this way, the early expansions caused by precipitation of ettringite in the small pores, where the capacity to accommodate ettringite is very limited, are neglected. As the expansions computed are the base of any future mechanical consideration, it is believed that this simplified expression is in contradiction with the sophisticated mechanical framework included in the advanced models. From this situation, the need to explore new ways to compute expansions, according to the degree of complexity and precision incorporated, is highlighted.

The objective of the present work is to present a diffusion - reaction model where a simplified approach of the chemical processes involved in external sulfate attack and the mechanical processes related to ettringite formation in the pores are considered. It is mainly based on the original proposal of Tixier \& Mobasher [11], which has been significantly modified by describing a new expression for the diffusion in a cracked porous media and through a new expression to compute the expansions. This new expression is based on a more realistic consideration of the porosimetry of concrete and on the introduction of a new ettringite formation rate $(\mathcal{F})$, which quantifies and distributes ettringite precipitation. By this approach, it is able to distinguish the different 
strain contributions from different pore sizes. In Fig. 1, a schematic diagram of the different processes considered in this model is depicted. Critical parameters of the numerical model developed will be recognized and established. Finally, expansions obtained by the new model will be compared to experimental data by Brown [13] and Ferraris et al. [14].

<Insert Fig. 1>

\section{2- DESCRIPTION OF THE CHEMO - TRANSPORT MODEL}

\section{1- CHEMICAL REACTIONS CONSIDERED}

In this model, it is considered that the deterioration process related to external sulfate attack is caused by the potential volume expansions associated to the chemical reactions of sulfate ions with the unreacted hydration compounds of the cement paste. Eqs. (1)-(4) describe the series of reactions considered to take place when the sulfate ions penetrate a cement based structure. This process is initiated by the reaction of sulfate ions $\left(\mathrm{SO}_{4}^{2-}\right)$ with calcium hydroxide $(\mathrm{CH})$ to form gypsum $\left(\mathrm{C} \bar{S} \mathrm{H}_{2}\right)$ (Eq. (1)).

$$
\mathrm{CH}+\mathrm{SO}_{4}^{2-} \rightarrow \mathrm{C} \overline{\mathrm{S}} \mathrm{H}_{2}+2 \mathrm{OH}^{-}
$$

Gypsum formation is considered as an intermediate phase that later may react with the aluminate phases to form secondary ettringite $\left(C_{6} A \overline{S_{3}} H_{32}\right)[11,15,16,17]$. Three calcium aluminates phases are considered as described in Eqs. (2)-(4): tricalcium aluminate 
$\left(C_{3} A\right)$, tetracalcium aluminate $\left(C_{4} A H_{13}\right)$ and monosulfate $\left(C_{4} A \bar{S} H_{12}\right)$. Note that no direct reactions between the sulfate solution ions and aluminate phases are contemplated, since it is assumed that all ingressing sulfates react first with calcium hydroxide to form gypsum.

$$
\begin{gathered}
C_{3} A+3 C \bar{S} H_{2}+26 H \rightarrow C_{6} A \overline{S_{3}} H_{32} \\
C_{4} A H_{13}+3 C \bar{S} H_{2}+14 H \rightarrow C_{6} A \overline{S_{3}} H_{32}+C H \\
C_{4} A \bar{S} H_{12}+2 C \bar{S} H_{2}+16 H \rightarrow C_{6} A \overline{S_{3}} H_{32}
\end{gathered}
$$

It is assumed that the expansions are caused only by the formation of secondary ettringite. The role of gypsum in the expansion mechanism is still far from clear [7], so in this study, the expansive nature of gypsum formation is not considered.

As previously proposed by Tixier \& Mobasher [11], and afterwards used by other researchers [4,17], Eqs. (2)-(4) are lumped in a single expression to simplify the numerical treatment (Eq. (5)). It should be noticed that by this simplification, one single chemical reaction rate will be used to define the kinetics of the reactions between the three calcium aluminates reactive phases and the gypsum formed.

$$
C A+q C \bar{S} H_{2} \rightarrow C_{6} A \overline{S_{3}} H_{32}
$$


In Eq. (5), $C A$ refers to the equivalent calcium aluminates (see Eq. (6)) and $q$ represents the stoichiometric weighted coeficcient of the sulfate phase (see Eq. (7)).

$$
\begin{gathered}
C A=\gamma_{1} C_{3} A+\gamma_{2} C_{4} A H_{13}+\gamma_{3} C_{4} A \bar{S} H_{12} \\
q=3 \gamma_{1}+3 \gamma_{2}+2 \gamma_{3}
\end{gathered}
$$

In Eqs. (6)-(7), the coefficients $\gamma_{\mathrm{i}}$ used are defined as the proportion of each aluminate phase to the total aluminate content.

\section{2- DIFFUSION - REACTION MODEL}

The deterioration processes defined by the chemical reactions take place depending on the availability of sulfate ions and calcium aluminates. Its concentrations are computed, for each time and space considered in the discretization of the structure, through a diffusion-reaction model based on the Fick's second law. It takes into account the diffusion of sulfate ions under a concentration gradient and its depletion due to gypsum formation.

Depending on the geometry of the structure under study, different formulations of the diffusion-reaction model are provided in Table 1. In this work, diffusion flux is classified as approximately unidirectional (typical in large elements with one dimension much smaller than the other two) and radial (typical in elements with small circular cross section, i.e. elements with two dimensions much smaller than the other one), where an intensification of the ingressing sulfate ions flux is produced as they progress 
towards inner layers. As underground and founding constructions are the most likely to be subjected to the external sulfate attack, in Table 1 it is also included the most representative underground structural typologies for both cases, although it is equally applicable to other typologies.

$\langle$ Insert Table 1>

One additional equation is also considered in both cases (Eq. (10)) to account for the calcium aluminates depletion to form ettringite.

$$
\frac{\partial C_{C A}}{\partial t}=-\frac{k C_{C A} C_{S O}}{q}
$$

Eqs. (8)-(10) denote $C_{S O}$ as sulfate concentration in the aggressive soil, $C_{C A}$ as aluminate concentration, $D$ as the effective diffusion coefficient, $k$ as the lumped rate of take-up sulfates and $R$ as the radius of the structure. The formulation used in the subsequent simulations is the one corresponding to radial diffusion fluxes, as the experimental results used to validate the present model were obtained from mortar bars with one dimension considerably larger than the other two.

\section{3- DIFFUSION COEFFICIENT OF SULFATE IONS}

Due to the chemical reactions triggered by the migration of sulfate ions throughout the capillary pores of concrete, the effective diffusivity of sulfate ions is highly dependent on the current stage of the degradation process. In this work, the diffusion coefficient is 
assumed to be dependent on the pore filling effect and on the damage state of the concrete matrix due to the expansive nature of ettringite. In this way, two parallel effects are computed for the calculation of the effective diffusion coefficient for each time and space considered: a decrease in diffusivity due to pore filling by ettringite and an increase of diffusivity due to cracking and spalling phenomena.

\subsection{1- PORE FILLING EFFECT}

The effective diffusivity of sulfate ions through concrete is decreased by the precipitation of ettringite in the pore system, as it constrains the transport paths for the ions diffusion. Different proposals may be found in the literature to consider the pore filling effect $[4,18,19]$. In this work, the hyperbolic function proposed by Idiart et al. [4] has been adopted, which yields comparable trends to the relationship proposed by Samson \& Marchand [19] and has the advantage to explicitly depend on the initial porosity $\left(\varphi_{0}\right)$. The diffusion coefficient is calculated according to Eq. (11).

$$
D=D_{\min }+\left(D_{0}-D_{\min }\right) \cdot \frac{e^{-\beta_{D}} \cdot \frac{\varphi}{\varphi_{0}}}{1+\left(e^{-\beta_{D}}-1\right) \frac{\varphi}{\varphi_{0}}}
$$

To simplify the mathematical representation of the diffusion phenomenon it is assumed that the system is saturated and all pores are accessible. In Eq. (11), $\varphi$ represents the updated porosity value, calculated by the Eq. (12). The term $\beta_{D}$ is a shape factor, adjusted in 1.5 as proposed by Idiart [16]. The initial capillary porosity is calculated by Powers model. Note that the hyperbolic function is defined within two limit values of 
the diffusion coefficient: $D_{0}$, representing the initial diffusivity (when $\frac{\varphi}{\varphi_{0}}=1$ ) and $D_{\text {min }}$, representing the minimum diffusivity yield when all the pore system is filled by ettringite (when $\frac{\varphi}{\varphi_{0}}=0$ ).

$$
\varphi=\operatorname{Max}\left[\varphi_{0}-\alpha_{s} C A^{\text {react }}, 0\right]
$$

In Eq. (12), the term $\alpha_{S} C A^{\text {react }}$ represents the porosity filled by ettringite formation, which, due to the chemical reactions considered, is computed by the calcium aluminates reacted. The parameter $\alpha_{s}$, which corresponds to a converting factor to obtain the volumetric expansion from the amount of aluminates reacted, is defined in Eq. (17).

\subsection{2- CRACKING EFFECT}

The volumetric expansion caused by ettringite formation can lead to cracking and spalling of the cementitious matrix when the stress produced by the expansions reaches the tensile strength of the material. This process increases the diffusivity of sulfate ions through the concrete matrix, as easier paths towards the inner layers may be found.

Traditionally, the increase in diffusivity due to cracking was related to a damage parameter $(w)$ defined by an isotropic damage model based on continuum damage mechanics $[3,11,15]$. In this work, a more direct and intuitive approach is presented, based on the idea that the loss of concrete strength is directly related to the cracking state of concrete, and hence, to the increase in diffusivity. It is important to remark that special types of failure could occur in elements with large or singular geometries 
subjected to large deformations. Such failures are related with the stress distribution inside the elements generated to maintain the strain compatibility and might occur outside the zone directly affected by the expansion. The simplified model proposed in this study do not account for this phenomenon, even though it is capable of simulating the repercussion of the damage in the diffusion mechanism in zones affected by the expansions. Despite that, the model should be valid as long as these special types of failure do not occur or if their influence on the diffusion mechanism remains small. In cases these conditions may not be guaranteed, more advanced simulation should be performed, for instance with coupled FEM.

This approach is based on the constitutive law of the material, depending on the elastic and the inelastic strain that may be present. The former corresponds to the elastic deformation of the material $\left(\varepsilon^{e}\right)$, and the latter corresponds to the contribution of cracking $\left(\varepsilon^{c}\right)$. In one dimension, the strain decomposition is represented by Eq. (13).

$$
\varepsilon=\varepsilon^{e}+\varepsilon^{c}
$$

To represent this behavior, a rheological model composed by an elastic spring that introduces the elastic strain $\left(\varepsilon^{e}\right)$ is coupled with a unit representing the contribution of the crack $\left(\varepsilon^{c}\right)$ [20]. As shown in Fig. 2, both unities transmit the same stress since they are coupled in series. 
To be able to distinguish the elastic and inelastic contribution of the total strain, a simplified constitutive law of the material has to be defined. In Fig. 3, the simplified constitutive law chosen is depicted and the nomenclature used in the subsequent definition of this new proposal is defined.

\section{<Insert Fig. 3>}

The ascending branch is defined by a linear elastic response of the concrete, based on Hook's law, until $\varepsilon^{\text {th }}$ is reached. In this region, only the elastic behavior is observed since the material is practically undamaged. When the stress reaches the tensile strength of the material $\left(f_{t}\right)$, the crack formation is initiated. After crack initiation, the softening non-linear law considered is the one proposed by Reinhardt and Hordijk [21] (Eq. 14).

$$
\sigma=f^{c}\left(\varepsilon^{c}\right) \equiv f_{t}\left\{\left[1+\left(\frac{c_{1} \varepsilon^{c}}{\varepsilon^{f}}\right)^{3}\right] \exp \left(-\frac{c_{2} \varepsilon^{c}}{\varepsilon^{f}}\right)-\exp ^{-c_{2}}\left(1+c_{1}^{3}\right) \frac{\varepsilon^{c}}{\varepsilon^{f}}\right\}
$$

As may be seen in Eq. (14), the softening law is completely defined by the material's uniaxial tensile strength $\left(f_{t}\right)$ and the strain at which the crack becomes stress-free $\left(\varepsilon^{f}\right)$. The physical significance of the strain $\varepsilon^{f}$ corresponds to a material where the strength loss is total, so the sulfate ions diffusivity reach the maximum value. The reference parameter for the definition of the exponential softening law is the fracture energy $G_{F}$, which represents the amount of energy released after cracking occurs. This is equivalent to the area below the stress-strain curve for a strain bigger than $\varepsilon^{\text {th }}$. In the present model, the value of $G_{F}$ may be estimated through Eq. (15), obtained through the integration of Eq. (14) depending on a characteristic cracking length $\left(l_{c h}\right)$ [21]. For the 
simulations performed in this paper, the value of $c_{1}$ and $c_{2}$ were defined respectively as 3 and 6.93, in accordance with the recommendation from Hordijk [21]. This entails fracture energy equal to $0.159 \mathrm{kN} / \mathrm{m}$ for a characteristic length of $26 \mathrm{~mm}$.

$$
\begin{aligned}
G_{F}=\int_{0}^{\varepsilon^{f}} l_{c h} & \sigma d \varepsilon \\
& =l_{c h} f_{t} \varepsilon^{f}\left\{\frac{1}{c_{2}}\left[1+6\left(\frac{c_{1}}{c_{2}}\right)^{3}\right]\right. \\
& -\left[\frac{1}{c_{2}}+c_{1}{ }^{3}\left(\frac{1}{c_{2}}+\frac{3}{c_{2}{ }^{2}}+\frac{6}{c_{2}{ }^{3}}+\frac{6}{c_{2}{ }^{4}}\right)\right. \\
& \left.\left.+\frac{1}{2}\left(1+c_{1}{ }^{3}\right)\right] \exp \left(-c_{2}\right)\right\}
\end{aligned}
$$

From a given linear strain, it is possible to distinguish the elastic and inelastic contributions of the total strain measured through the material constitutive law defined in Fig. 3. As the degree of concrete strength loss (i.e. the degree of cracking) is defined by the inelastic contribution, only this part of the total strain causes the formation of cracks that modify the diffusivity. Therefore, to consider the influence of cracking on sulfates diffusivity, a relation between inelastic strains and diffusion coefficient must be defined.

In this regard, according to the strain level reached, uncracked, cracked and fully cracked regions are defined (Fig. 4). The uncracked region covers the domain where the inelastic strain is equal to zero since the tensile strength of the material has not been reached and only elastic strain is observed. In this domain the diffusivity is not increased. On the other hand, in the cracked zone, the strain reached is high enough to develop inelastic contributions. Consequently, the diffusivity is increased by the cracking until a maximum diffusivity value is reached $\left(D_{\max }\right)$. This maximum value, 
corresponds to the strain where the concrete strength loss is total. As this new proposal is based on the idea that concrete strength loss is directly related to the cracking state of the material, the slope of this curve will be defined by the tangent of the softening curve defined in the material constitutive law (see Fig. 3). Once the maximum diffusivity value is reached, it will remain constant for further deformations.

\section{<Insert Fig. 4>}

In the literature, no agreement was found in the definition of the upper bound $\left(D_{\max }\right)$ of the function depicted in Fig. 4, representing the diffusivity of sulfate ions in a medium that has lost all its capability to carry stresses. Since few experimental studies may be found on the diffusion of sulfate ions through cracked concrete specimens, results from chloride migration test are commonly used to quantify this parameter. Although in this case more results are available, different increase factor of chloride diffusivity are suggested. Gerard \& Marchand [22] quantify the increase factor from 2 to 10 , whereas Sahmaran et al. [23] assessed increasing factors of 200 for a crack width of $400 \mu \mathrm{m}$. According to the results of Djerbi et al. [24], Idiart et al. [4] proposed that in a completely cracked medium, the diffusion coefficient tends to reach the diffusivity in free solution.

The value adopted in this paper for the upper bond diffusivity $\left(D_{\max }\right)$ is the free solution diffusivity, which Gerard \& Marchand [22] quantified as $10^{-9} \mathrm{~m}^{2} / \mathrm{s}$. Although the authors acknowledge that the ions are able to move freely only within cracks, given the lack of agreement in the quantification of an upper bound for the diffusion coefficient, it was decided to fix this value at the diffusivity in free solution. Notice that 
other considerations based on reliable experimental results may also be used in the model if appropriate.

\section{3- MODELING VOLUMETRIC EXPANSIONS}

Amongst the several mechanisms that have been suggested in the literature to explain how the precipitation of ettringite leads to expansion, two theories stand as the most discussed [25]. In the first of them, expansion are a result of the additional volume generated by ettringite formation (e.g. $[3,11,17,26])$. In the second of them, expansions are caused by the crystallization pressure exerted on the pore walls due to the formation of ettringite from supersaturated solution within small pores (e.g. [25,27]). The latter theory is supported by recent publications [25,28,29], placing this approach as the most plausible theory [29]. Nevertheless, the use of the volume increase approach has persisted in the literature even though it lacks an explanation for the force needed to cause the expansions. The reason may be found in the inherent difficulties associated to the modelization of the crystallization pressure theory, where pores cannot be treated as independent elements and interconnectivity between them must be taken into account during the crystallization process.

Due to the need of simplifying the computational modelization, the first approach is used here, assuming that the volumetric strain is the result of the volume change associated with the chemical reactions. A new way to compute expansions is presented, by which it is possible to obtain the different strain contributions provided by each pore size. This new proposal is more representative of the real situation and is able to reflect some important effects described by the crystallization pressure theory since it takes 
into account the greater contribution of the small pores in the overall expansion. In fact, the new model proposed opens up the possibility of combining both theories given that the crystallization pressure may be used as an indirect qualitative reference to define the buffer sulfate concentration depending on the pore size.

Expansions are defined by the previously mentioned chemical reactions considered in the model. In this work, ettringite is considered as the only reaction product governing the expansions of the structure. As mentioned before, the possible expansion due to gypsum formation is neglected. The additional volume generated by the reaction products is calculated by using stoichiometric constants [26]. As described in Eq. (5), ettringite formation is directly defined by the calcium aluminates reacted, thus the volumetric strain can be calculated by the amount of calcium aluminates reacted. According to the three aluminate phases considered in the present model, the additional volume can be calculated as indicated in Eq. (16).

$$
\frac{\Delta V}{V}=\sum_{1}^{3} \frac{\Delta V_{i}}{V_{i}} \frac{M_{i}}{\rho_{i}} \frac{C_{i}}{\sum_{1}^{3} C_{i}} \cdot C A^{\text {react }}
$$

The term $\Delta V_{i} / V_{i}$ is the volumetric change of each reaction in Eqs. (2)-(4), whose values are listed in Table 2. The weight of each aluminate phase is quantified by the term $C_{i} / \sum_{1}^{3} C_{i}$, where $C_{i}$ correponds to the total concentration of each aluminate phase considered. The term $M_{i} / \rho_{i}$ corresponds to the molar volume of each aluminate phase and $C A^{\text {react }}$ is the total concentration of calcium aluminates reacted to form ettringite. 
According to the notation previously used to define the decrease of diffusivity due to pore filling (Eq. (12)), $\alpha_{s}$ can be expressed as in Eq. (17).

$$
\alpha_{s}=\sum_{1}^{3} \frac{\Delta V_{i}}{V_{i}} \frac{M_{i}}{\rho_{i}} \frac{C_{i}}{\sum_{1}^{3} C_{i}}
$$

<Insert Table 2>

Whether expansion actually occurs or not, will depend on the capacity of the pore structure to accommodate ettringite as well as on the capacity of the matrix to resist expansive stresses [11,26,31]. All recent models based on the additional volume generated approach use the expression presented by Tixier \& Mobasher (Eq. 18) [11].

$$
\varepsilon_{\mathrm{v}}=\frac{\Delta V}{V}-f \varphi_{0}
$$

The second term $\left(f \varphi_{0}\right)$ represents the fraction of the concrete initial porosity that has to be filled prior expansion starts, usually fixed in a range between 5\%-40\% [11]. Note that the buffer coefficient is linearly applied to the overall ettringite formation and the overall porosity is considered without differentiating the size of the pores. This is equivalent to say that in the precipitation process, the amount of ettringite inside each pore is proportional to its volume. This situation is represented in Fig. 5, where all the complexity of the concrete porosimetry is reduced to a single pore, where all ettringite precipitates in. Consequently, it is indirectly assumed that the expansion occurs 
simultaneously in all pores, regardless of their size. However, this contradicts the observation from Scherer [27], which showed an inverse relationship between the pressure exerted and the size of the pore. Therefore, crystals growing in small pores, with a low capability to accommodate ettringite, are more likely to generate important pressures to the pore wall.

\section{<Insert Fig. 5>}

To account for these phenomena, a new simplified approach is presented. It considers an ettringite formation rate $(\mathcal{F})$ which quantifies and distributes the ettringite within the different pore sizes considered in the discretization of the concrete porosimetry. The formation rate $\mathcal{F}$ is defined in Eq. (19) as a constant parameter within each cell and time step considered.

$$
\mathcal{F}=\frac{Q_{T}}{S_{T}}
$$

The term $Q_{T}$ is the volume of ettringite precipitated, described in Eq. (20). The term $S_{T}$ is the total pores surface, described in Eq. (21). In Fig. 6, a schematic representation of those parameters is depicted.

$$
\begin{gathered}
Q_{T}=\frac{\Delta V}{V} V_{\text {cell }} \\
S_{T}=\sum_{i=1}^{n} S_{i}=\sum_{i=1}^{n} n_{i} 4 \pi R_{i}^{2}
\end{gathered}
$$


The term $R_{i}$ is the radio of the pore size $i$ and $V_{\text {cell }}$ corresponds to the total volume of the cell under consideration. The term $n_{i}$ is the number of pores of each pore size, as described in Eq. (22).

$$
n_{i}=\frac{V_{\text {cell }} \varphi_{0} X_{\mathrm{i}}}{4 / 3 \pi R_{i}^{3}}
$$

As can be seen in Eq. (22), pores are considered to have a round shape. The only parameter not yet defined is $X_{\mathrm{i}}$, which represents the pore ratio of each size. Both parameters $R_{i}$ and $X_{\mathrm{i}}$ can be defined by a pore size distribution diagram, commonly obtained through mercury intrusion porosimetry (MIP). From the porosimetry distribution, the curve obtained can be discretised into a finite number of pore sizes. Once the discretization is defined, $X_{i}$ is obtained through a simple area ratio as defined in Eq. (23). The equivalent radius $R_{i}$ is estimated as the mean radius for each pore size considered.

$$
X_{i}=\frac{A_{\mathrm{i}}}{A_{T}}
$$

This procedure is represented in Fig. 7, which depicts the porosimetry distribution of a CEM I $42.5 \mathrm{R} / \mathrm{SR}$ concrete, with a simple discretization of the pores into three pore 
sizes according to the capillary pore classes defined by Mindess [32]. Each $\mathrm{X}_{\mathrm{i}}$ can be obtained by a simple area ratio between the colored areas.

\section{<Insert Fig. 7>}

Once the formation rate term $\mathcal{F}$ is defined, the criteria by which the amount of ettringite formed is distributed within the different pore sizes is assessed. The crystallization process of ettringite is a complex dynamic phenomenon where the effects of the interconnectivity of the pore network play an essential role. However, due to the simplifying assumptions adopted for computational modelization, these effects are not taken into account explicitly such as in the crystallization theory. From Eq. (19), the volume of ettringite precipitated in each pore of size $i$ can be defined as the ettringite formation rate $\mathcal{F}$ present in the cell multiplied by the corresponding internal surface of the pore $S_{i}$. Therefore, as described in Eq. (24), pores with bigger surface, will receive more volume of ettringite.

$$
Q_{i}=\mathcal{F} S_{i}
$$

However, the initiation of the expansion is not related with the total amount of ettringite but with the proportion of the pore that is filled. Concerning the numerical model, the amount of ettringite precipitated in each pore size is not relevant. That is why the above mentioned equation is divided by its corresponding pore volume $V_{i}$. 


$$
\frac{Q_{i}}{V_{i}}=\mathcal{F} \frac{S_{i}}{V_{i}}
$$

In Eq. (25), $Q_{i} / V_{i}$ is the pore volume ratio filled by ettringite. Through this expression, it can be seen that small pores, with a high $S_{i} / V_{i}$ ratio, are filled faster than bigger pores. Hence, despite small pores receive a lower amount of ettringite compared to bigger pores (Eq. (24)), they are filled faster, due to its higher $S_{i} / V_{i}$ ratio (Eq. (25)).

Finally, the volumetric expansion $\left(\varepsilon_{v}\right)$, is obtained by considering the contribution to the volumetric expansion of each pore size $\left(\varepsilon_{i}\right)$ :

$$
\begin{gathered}
\varepsilon_{i}=\operatorname{Max}\left[\frac{Q_{i}}{V_{i}}-f_{i}, 0\right] \frac{V_{i}}{\sum V_{i}} \\
\varepsilon_{v}=\varepsilon_{1}+\varepsilon_{2}+\cdots+\varepsilon_{n}
\end{gathered}
$$

It should be noted that through the new equation to compute expansions (Eq. 26), it is possible to define a different buffer coefficient $\left(f_{i}\right)$ for each pore size. Therefore, it is able to consider the lower capability of small pores to accommodate ettringite without exerting pressure to the pore walls. However, in the model proposed in this paper, the influence of the interconnectivity of the pore network in the pressure stability and in the ettringite precipitation is not taken into account explicitly such as in the crystallization theory. This effect is simulated indirectly by means of a minimum sulfate concentration required for expansion, which varies with the pore size. 
This new proposal, based on the simplified approach that considers expansions as the result of the additional volume generated by ettringite formation, is not only applicable to the external sulfate attack phenomenon, but to every single deterioration process associated to the precipitation of expansive reaction products within the pore network.

\section{4- PARAMETRIC STUDY}

In order to detect the critical parameters of the numerical model developed, a parametric analysis was conducted in which five parameters were considered. As shown in Fig. 8, the influence of each parameter is evaluated by the aluminates depletion profile. The advance of the reaction front is evaluated at three different points to define its amplitude. This provides information regarding which is the governing process: the diffusion or the chemical reaction.

\section{<Insert Fig. 8>}

The parameters considered and its ranging values are listed on Table 3 . The ranges were defined according to realistic values found in the literature. The parameter under study is varied within a range defined by the maximum and minimum values while the other parameters are fixed in its reference values. Total time simulated is fixed at 5 years. The porosimetry distribution used in this study is based on the MIP results obtained by Abdelkader for a 28 days concrete CEM I 42.5 R/SR [33]. The curve is discretized in 4 pore sizes and the corresponding pore ratios are obtained through Eq. (23). In Table 4, values of $X_{\mathrm{i}}$ and $R_{i}$ used during the simulations are listed. The buffer coefficients $\left(f_{i}\right)$ are fixed at the same value in all pore sizes considered for each simulation. 
$<$ Insert Table 3>

<Insert Table 4>

More than 75 simulations were carried out in the parametric study in order to detect which parameters influence more the response of the model. According to the criteria defined to evaluate the influence of the different parameters considered, the reaction front variation is evaluated. To quantify it, the variation of the curve which corresponds to the $50 \%$ reacted is assessed within the range under study. Table 5 shows the ranked list that has resulted from this analysis. The sulfate concentration in the aggressive soil $\left(C_{S O}\right)$ and the lumped rate of take-up sulfates $(k)$, resulted to be the most influencing parameters on the reaction front. On the other hand, changes in the initial diffusivity $\left(D_{0}\right)$ and calcium aluminates concentration $\left(C_{C A}\right)$ do not affect significantly the reaction front.

\section{<Insert Table 5>}

Fig. 9a shows the variation of the reaction front for different values of the sulfate concentration $\left(C_{S O}\right)$. A uniform increase in the reaction front is depicted when the sulfate concentration increases within the range defined. Regarding the front amplitude, it can be seen that it remains constant during the whole range under study. This behavior means that variations in the sulfate concentration do not modify the weight of the two processes involved (diffusion and chemical reactions). 
In Fig. 9b, the influence of the lumped rate of take-up sulfates $(k)$ is evaluated. In this case, the reaction front shows an important increment for values between the minimum $\left(10^{-9} \mathrm{~m}^{3} / \mathrm{mol} \cdot \mathrm{s}\right)$ and the reference $\left(10^{-8} \mathrm{~m}^{3} / \mathrm{mol} \cdot \mathrm{s}\right)$. This important increment is related to crack initiation, which results into an amplification of the effective diffusivity. For values above the reference, the increment in the reaction front is more moderate and tends to stabilize. As expected, the front amplitude shows a high influence on the lumped rate $k$. For low values of $k$, the front amplitude increases considerably, showing that the diffusion process is gaining prominence in comparison to the chemical reaction process. While for high values of $k$, the front amplitude is considerably reduced. This reduction can be explained by the fact that with an increase of the reaction rate, a higher number of diffusive sulfate ions are involved in chemical reactions as they progress towards inner layers, so they are no longer available for the diffusion process.

\section{<Insert Fig. 9>}

The influence of the initial diffusivity $\left(D_{0}\right)$ on the reaction front is depicted in Fig. 9c. It can be seen that variations in diffusivity between $10^{-13} \mathrm{~m}^{2} / \mathrm{s}$ to $10^{-11} \mathrm{~m}^{2} / \mathrm{s}$ do not affect significantly the penetration of the reaction front. It is important to note that the influence gain prominence when the $5 \%$ reacted front, which is the most representative of the diffusion process, is evaluated. However, the $95 \%$ reacted front, more representative of the chemical reaction process, is barely affected by the initial diffusivity. As a result, the front amplitude shows an important increase within the range under study. Reflecting the fact that this parameter has more influence on the diffusion process rather than on the chemical one. 
In Fig. 9d the influence of calcium aluminates concentration $\left(C_{C A}\right)$ is represented. This parameter showed the smallest influence on the reaction front amongst all the parameters considered. It should be remembered that in this study, the influence on the expansions profile, where the aluminates concentration is a key parameter, is not evaluated. Surprisingly, the front amplitude shows a slight increment with the increase of calcium aluminates. This fact means that the increase on the reaction front when the aluminates concentration increases is caused, to a greater extend, by the diffusion process.

Finally, the buffer coefficient $\left(f_{i}\right)$ is evaluated. This parameter is placed in a different set of figures because in this case, the results obtained by the consideration of different pore sizes are also compared to the ones obtained by the traditional approach, which does not differentiate the size of the pores. In Fig. 10a the influence on the reaction front is depicted. As expected, the reaction front decreases as the buffer capacity increases, since more ettringite can be accommodated in the pores. However, the uniform slope of this tendency was not so expected. To understand this behavior, in Fig. 10b the results obtained are compared to the traditional unified buffer coefficient approach. For clarity, only the curves corresponding to the $50 \%$ reacted front are depicted in both cases. It can be seen that by considering different pore sizes, the reaction front always reaches higher values. Therefore, the influence of the buffer coefficient is considerably reduced. This behavior may be explained by the fact that through the consideration of different pore sizes, expansions start to compute earlier, as small pores have a limited capacity to accommodate ettringite crystals. This expansions will trigger the increase in diffusivity due to cracking since earlier stages, so the reaction front will penetrate more into inner 
layers. As can be seen in Fig. 10b, the differences between the two approaches are more relevant for high values of the buffer capacity.

<Insert Fig. 10>

\section{5- VALIDATION}

Experimental results by Brown [13] and Ferraris et al. [14] are used to validate the expansions obtained through the model developed in this paper. The potential expansion calculated with the models proposed here are the direct result of the external sulfate attack. However, depending on the boundary conditions and on the size of the specimen, this expansion attributed to the chemical reactions may be reduced due to the compatibility of strain of adjacent elements that compose the cross section. In other words, the mechanical behavior of the element affects the resulting macroscopic expansion experienced by the cross section. In order to validate the model developed to predict the potential expansion due to the chemical reactions using experimental data from the literature, the mechanical behavior has to be considered. In this context, the macroscopic expansions of the simulated specimens are obtained through a typical sectional analysis, which considers that plane sections remain plane after the deformation (due to compatibility of strain).

Since the diffusion process is much slower than the chemical reactions, the external sulfate attack is initially limited to a region close to the external surface of the specimen. Consequently, two zones may be defined in the cross section: a more external layer affected by the attack and an inner core that has not been yet affected by it. Due to the 
compatibility of strains, the inner core contributes to stiffen the specimen or structural element, hence restraining the expansions experienced by the external layer. This situation is simulated through Eq. (28), which provides the resultant strain $\left(\Delta \varepsilon_{\text {section }}\right)$ observed at the instant $t_{n+1}$.

$$
\Delta \varepsilon_{\text {section }}\left(t_{n+1}\right)=\frac{\int_{\text {Area }} E\left(t_{n}, x\right) \cdot \Delta \varepsilon_{\text {exp }}\left(t_{n+1}, t_{n}, x\right) d \text { Area }}{\int_{\text {Area }} E\left(t_{n}, x\right) \text { dArea }}
$$

In this equation, the term $E\left(t_{n}, \mathrm{x}\right)$ represents the elastic modulus of the material at a certain time and $\Delta \varepsilon_{\text {exp }}$ corresponds to the expansion increment resulting from the external sulfate attack in the time step and position calculated with the model proposed in this work. It is important to remark that the term Area indicated in Eq. (28) stands for the total cross section of the specimen, not only the directly affected by the reaction. Notice that the integral in the numerator includes the term $\Delta \varepsilon_{\text {exp }}$, which will be 0 for zones without expansion due to the external sulfate attack. These zones, however, contribute for the integral in the denominator. Consequently, $\Delta \varepsilon_{\text {section }}$ will be smaller than the maximum $\Delta \varepsilon_{\text {exp }}$ estimated at a certain time. For the assessment of the stress level, the difference between the total strain and the non-mechanical strain (i.e. $\Delta \varepsilon_{\text {exp }}$ ) is considered according with equation Eq. (29). As expected, $\Delta \sigma_{c}$ will assume negative values in zones highly affected by the attack, indicating the presence of compressive stresses. On the contrary, in zones from the inner core not affected by the expansion $\left(\Delta \varepsilon_{\text {exp }}=0\right)$, the term $\Delta \sigma_{c}$ will be positive, indicating the existence of tensile stresses.

$$
\Delta \sigma_{c}\left(t_{n+1}, x\right)=E\left(t_{n}, x\right) \cdot\left[\Delta \varepsilon_{\text {section }}\left(t_{n+1}, x\right)-\Delta \varepsilon_{\text {exp }}\left(t_{n+1}, x\right)\right]
$$


It is also necessary to take into account that a very high stress is generated by the external sulfate attack in a small region close to the surface of the sample, since the diffusion process is much slower than the chemical reactions. This tends to produce a layered damage of the sample, which has been described by many authors that tested mortar prisms exposed to an external sulfate attack. To account for this in the mechanical model, a simplified consideration of the damage was introduced. Damage in the compressed zone of the specimens is modelled through a degradation of the elastic modulus, initiating a plastic behavior of the material for stresses higher than $80 \%$ of the compressive strength.

Notice that the mechanical model used consider several simplifications. In fact, more advanced mechanical models should be selected for the analysis of complex elements or structures subjected to cracking and nonlinearities. In this context, the new approach proposed to estimate the potential strain due to the chemical reactions represents the main contribution of this work since it could be implemented in other types of mechanical models.

\section{1- VALIDATION BASED ON EXPERIMENTAL RESULTS BY BROWN}

In 1981, Brown performed a series of experimental tests to investigate how a controlled environment accelerates the rate of sulfate attack [13]. The linear expansions were measured for mortar bars immersed in a $352 \mathrm{mmol}$ of sulfates/liter solution while maintaining the solution $\mathrm{pH}$ at 10 . Mortar bars were cast at $\mathrm{w} / \mathrm{c}=0.6$ using a high $\mathrm{C}_{3} \mathrm{~A}$, $14 \%$, type I cement. Lacking the experimental value for the lumped rate of take-up of sulfates and the fraction of the pore that can be filled without causing expansions, for 
the time being, $k$ and $f_{i}$ are considered as fitting parameters of the model. No information regarding the porosimetry distribution was available, so realistic values for the mortar used were adopted. The values of the main parameters for fitting the data are shown in Table 6. Other parameters used in the computations were: $\mathrm{D}_{\min }=\mathrm{D}_{0} / 10$ [22], $\mathrm{D}_{\max }=1000 \mathrm{D}_{0}[16], \varepsilon_{f}=0.005, \mathrm{c}_{1}=3[21], \mathrm{c}_{2}=6.93[21], \mathrm{f}_{\mathrm{t}}=2.89 \mathrm{~N} / \mathrm{mm}^{2}, \mathrm{f}_{\mathrm{c}}=25 \mathrm{~N} / \mathrm{mm}^{2}$, $\mathrm{E}_{28}=28000 \mathrm{~N} / \mathrm{mm}^{2}, \mathrm{w} / \mathrm{c}=0.6, \beta_{D}=1.5[16], \mathrm{q}=3, \mathrm{R}_{1}=8 \mathrm{~nm}, \mathrm{R}_{2}=15 \mathrm{~nm}, \mathrm{R}_{3}=30 \mathrm{~nm}, \mathrm{R}_{4}=50$ $\mathrm{nm}, \mathrm{R}_{5}=0.1 \mu \mathrm{m}$ and $\Delta \mathrm{V} / \mathrm{V} \cdot \mathrm{m}=0.000170 \mathrm{~m}^{3} / \mathrm{mol}$ (for monosulfate). The number of space intervals and time steps considered were 50 and $50 \mathrm{~s}$, respectively.

\section{<Insert Table 6>}

The experimental results were contrasted with the outputs of the model developed and compared with the evolution of longitudinal expansions obtained by the model of Tixier \& Mobasher [34]. As may be seen in Fig. 11, the shape of the curve provided by the model from the authors is not able to fit accurately the experimental data. The reason for this may be found into the consideration of the buffer effect from the porosity and the influence of the mechanical behavior. By considering a single buffer coefficient applied to the overall porosity, all ettringite formed during early stages of the simulation is neglected since it is absorbed by the pores. Consequently, a null expansion is estimated initially. However, once this buffer capacity is filled, the additional ettringite precipitated leads to an increase of internal stress and expansion. After a critical tensile stress is reached, failure occurs and part of the stress is redistributed, producing an extra expansion. From this point on, an abrupt increase of strains is observed as a result of a combination of a chemical phenomenon (the additional formation of ettringite) and a mechanical phenomenon (release of stress due to cracking). This combined effect may 
explain the sudden increase in the estimations provided by Tixier and Mobasher [34] in Fig. 11. On the contrary, the estimations provided by the model developed here show a much better fit throughout the 60 days of the simulation. This is the result of the new approach proposed for the buffer capacity of different pore sizes.

\section{<Insert Fig. 11>}

Fig. 12a shows the fraction filled at the end of the simulation for each pore size considered. As expected, as the pore size decreases the pore fraction filled increases, being the Pore Size 1, corresponding to the smallest radio $\mathrm{R}_{1}=8 \mathrm{~nm}$, the one that has resulted in a higher fraction filled. In Fig. 12b, the contribution of each pore size into the total volumetric expansion is represented. As the strains are calculated by the pore fraction filled (Eq. 26), the contribution from small pores to the overall expansion could be larger than that from large pores. In the current simulation, pores of radio $50 \mathrm{~nm}$ and $100 \mathrm{~nm}$ do not contribute to the expansions. This fact reflects the phenomenon described by the crystallization pressure theory, in which crystals growing in small pores (of order of tens of nanometers) are more likely to generate significant expansive pressure [29]. In a recent publication by Müllauer [35], it was quantified that the formation of ettringite in small pores (10 to $50 \mathrm{~nm}$ ) generates a stress of about $8 \mathrm{MPa}$ that exceeds the tensile strength of the binder matrix and is responsible for damage. The same author suggests that bigger pores are not able to generate enough crystallization pressure to exceed the tensile strength of the matrix. Although the model developed here reproduces this behavior, the results cannot be quantitatively compared to the ones obtained through a crystallization pressure model since the expansions are computed solely from the additional volume generated. 
<Insert Fig. 12>

\section{2- VALIDATION BASED ON EXPERIMENTAL RESULTS BY FERRARIS ET} AL.

Additional validation of the expansion profile is performed using experimental data from Ferraris et al. [14]. Mortar prisms $25 \mathrm{~mm}$ x $25 \mathrm{~mm}$ x $276 \mathrm{~mm}$ were immersed in $5 \%$ solution of sodium sulfate. The $\mathrm{pH}$ of the solution was maintained at 7 until disintegration of the specimen occurred. The tests used a cement with a $\mathrm{C}_{3} \mathrm{~A}$ content at $12.8 \%$ and a w/c ratio of 0.485 . Changes in length of each specimen were monitored. No information regarding the porosimetry distribution was available, so realistic values for the mortar used were adopted. For consistency, same pore sizes as in the validation based on the experimental results by Brown are considered. As in the previous validation, no experimental values for the parameters $k$ and $f_{i}$ are provided. This scenario force to consider $k$ and $f_{i}$ as fitting parameters of the model. The values of the main parameters for fitting the data are shown in Table 7. Other parameters used in the computations were: $\mathrm{D}_{\min }=\mathrm{D}_{0} / 10$ [22], $\mathrm{D}_{\max }=1000 \mathrm{D}_{0}[16], \varepsilon_{f}=0.005, \mathrm{c}_{1}=3$ [21], $\mathrm{c}_{2}=6.93[21], \mathrm{f}_{\mathrm{t}}=2.89 \mathrm{~N} / \mathrm{mm}^{2}, \mathrm{f}_{\mathrm{c}}=25 \mathrm{~N} / \mathrm{mm}^{2}, \mathrm{E}_{28}=28000 \mathrm{~N} / \mathrm{mm}^{2}, \mathrm{w} / \mathrm{c}=0.485, \beta_{D}=1.5$ [16], $\mathrm{q}=3, \mathrm{R}_{1}=8 \mathrm{~nm}, \mathrm{R}_{2}=15 \mathrm{~nm}, \mathrm{R}_{3}=30 \mathrm{~nm}, \mathrm{R}_{4}=50 \mathrm{~nm}, \mathrm{R}_{5}=0.1 \mu \mathrm{m}$ and $\Delta V / \mathrm{V} \cdot \mathrm{m}=0.000170$ $\mathrm{m}^{3} / \mathrm{mol}$. The number of space intervals and time steps considered were 40 and $75 \mathrm{~s}$, respectively. 
In Fig. 13, the longitudinal expansions obtained by the model developed in the present study are compared with the expansions obtained by Tixier \& Mobasher [34] and experimental measurements from Ferraris et al. [14]. The prediction provided by the model of Tixier \& Mobasher presents a good fit until the late stage of the simulation, where the last point measured during the experimental campaign is not fitted by the model. The prediction provided by the approach presented in this paper shows a good fit throughout the 120 days of the simulation.

<Insert Fig. 13>

The pore fraction filled at the end of the simulation, and the contribution of each pore size into the volumetric expansion are depicted in Fig. 14a and 14b, respectively. As in the previous validation, as the pore size decreases the pore fraction filled increases, according to the higher surface/volume ratio present in small pores. Filled percentages reached $100 \%$ for the smallest pore, and almost $12 \%$ for the biggest one at the end of the simulation. The behavior depicted in Fig. 14b, where pores from $8 \mathrm{~nm}$ to $30 \mathrm{~nm}$ contribute to the total strain, is in accordance with the phenomenon described by the crystallization pressure theory $[29,35]$.

<Insert Fig. 14>

\section{6- CONCLUSIONS}

The following conclusions may be derived from the present study. 
(1)- A new expression for the consideration of the diffusion in a cracked porous media has been proposed based on the constitutive law of the material. It allows a more direct and intuitive approach since it does not require the definition of any damage parameter such as the one used by isotropic damage models based on continuum damage mechanics. In this case, the damage is considered indirectly, thus making the estimations simpler.

(2)- A new approach to compute expansions is presented, based on a more realistic consideration of the concrete porosimetry. The described approach allows the consideration of different filling rates and capacities to accommodate expansive product for each pore size considered. These features, already considered in models based on crystallization pressure theory, were not possible to be considered in models based on the simplified theory of the volume increase approach until now. The authors believe that through this path, it will be possible to consistently model the expectable effects of the crystallization pressure theory through the simplified volume increase approach.

(3)- Referring to the parametric analysis, the sulfate concentration in the aggressive soil and the lumped rate of take-up sulfates, resulted to be the most influencing parameters on the reaction front. On the other hand, changes in the initial diffusivity and calcium aluminates concentration do not affect significantly the reaction front.

(4)- Expansions obtained by the new model are in good agreement with experimental data by Brown [13] and Ferraris et al. [14], suggesting that the proposed model is able to predict its behavior. As described by the crystallization pressure theory, a bigger contribution of small pores into the total strain is observed in the simulations [29]. More 
advanced mechanical models capable of accounting for the failure of the specimens should be selected for the analysis of more complex elements or structures.

\section{Acknowledgments}

Support from the Spanish Ministry of Economy and Competitiveness through research project BIA2010-20913-C02-02 is greatly acknowledged. Dr. I. Segura is supported by the postdoctoral Juan de la Cierva program of the Spanish Ministry of Economy and Competitiveness.

\section{References}

[1] EUROCONSTRUCT Conference, VTT, Buildecon. Construction in Europe breakdown in 2012 .

[2] O.S. Baghabra Al-Amoudi, Attack on plain and blended cements exposed to aggressive sulfate environments, Cem. Concr. Compos. 24 (2002) 305-316.

[3] S. Sarkar, S. Mahadevan, J.C.L. Meeussen, H. van der Sloot, D.S. Kosson, Numerical simulation of cementitious materials degradation under external sulfate attack, Cem. Concr. Compos. 32 (2010) 241-252.

[4] A.E. Idiart, C.M. López, I. Carol, Chemo-mechanical analysis of concrete cracking and degradation due to external sulfate attack: A meso-scale model,» Cem. Concr. Compos. 33 (2011) 411-423.

[5] C. Ayora, S. Chinchón, A. Aguado, F. Guirado, Weathering of iron sulfides and concrete alteration: Thermodynamic model and observation in dams from central Pyrenees, Spain, Cem. Concr. Res. 28 (9) (1998) 1223-1235. 
[6] A. Campos, Análisis numérico de presas de hormigón bajo acciones expansivas, Phd Thesis, Universitat Politécnica de Catalunya, Barcelona, 2012.

[7] A. Neville, The confused world of sulfate attack on concrete, Cem. Concr. Res. 34 (2004) 1275-1296.

[8] P.K. Mehta, Sulfate attack on concrete - a critical review, Mater. Sci. Concr. III, Am. Ceram. Soc., Westerville, 1992, pp. 105-130.

[9] J.P. Skalny, I. Odler, J. Marchand, Sulfate Attack on Concrete, Spon, London, 2001.

[10] M. Collepardi, A state-of-the-art review on delayed ettringite attack on concrete, Cem. Concr. Compos. 25 (2003) 401-407.

[11] R. Tixier, B. Mobasher, Modeling of Damage in Cement-Based Materials Subjected to External Sulfate Attack. I: Formulation, J. Mater. Civ. Eng. 15 (2003) 305313.

[12] B. Bary, Simplified coupled chemo-mechanical modeling of cement pastes behavior subjected to combined leaching and external sulfate attack, Int. J. Numer. Anal. Meth. Geomech. 32 (2008) 1791-1816.

[13] P.W. Brown, An evaluation of the sulfate resistance of cements in a controlled environment, Cem. Concr. Res. 11 (1981) 719-727.

[14] C.F. Ferraris, J.R. Clifton, P.E. Stutzman and E.J. Garboczi, Mechanisms of degradation of portland cement-based systems by sulfate attack, in: K.L. Scrivener and J.F. Young (Eds), Mechanisms of chemical degradation of cement-based systems, E\&FN Spon, London, 1997, pp. 185-192.

[15] M. Basista, W. Weglewski, Chemically assisted damage of concrete: a model of expansion under sulfate attack, Int. J. Damage Mech. 18 (2008) 155-175. 
[16] A.E. Idiart, Coupled analysis of degradation processes in concrete specimens at the meso-level, PhD Thesis, Universitat Politécnica de Catalunya, Barcelona, 2009. [17] X.B. Zuo, W. Sun, C. Yu, Numerical investigation on expansive volume strain in concrete subjected to sulfate attack, Constr. Build. Mater. 36 (2012) 404-410. [18] P.N. Gospodinov, R.F. Kazandjiev, T.A. Partalin, M.K. Mironova, Diffusion of sulfate ions into cement stone regarding simultaneous chemical reactions and resulting effects, Cem. Concr. Res. 29 (1999) 1591-1596.

[19] E. Samson, J. Marchand, Modeling the transport of ions in unsaturated cement based materials, Comput. Struct. 85 (2007) 1740-56.

[20] M. Jirásek, Damage and Smeared Crack Models, in: G. Hofstetter and G. Meschke (Eds.), Numerical Modeling of Concrete Cracking, CISM, Udine, 2011, pp. 149.

[21] D.A. Hordijk, Local Approach to Fatigue of Concrete, Phd Thesis, Delft University of Technology, Delft, 1991.

[22] B. Gérard, J. Marchand, Influence of cracking on the diffusion properties of cement-based materials. Part I: Influence of continuous cracks on the steady-state regime, Cem. Concr. Res. 30 (2000) 37-43.

[23] M. Sahmaran, M. Li, and V.C. Li, Transport Properties of Engineered Cementitious Composites under Chloride Exposure, ACI Mater. J., V. 104, No. 6, (2007) 303-310.

[24] A. Djerbi, S. Bonnet, A. Khelidj, V. Baroghel-bouny, Influence of traversing crack on chloride diffusion into concrete, Cem. Concr. Res. 38 (2008) 877-883.

[25] W. Kunther, B. Lothenbach, K. Scrivener, On the relevance of volume increase for the length changes of mortar bars in sulfate solutions, Cem. Concr. Res. 46 (2013) 23-29. 
[26] J.R. Clifton, J.M. Pommersheim, Sulfate attack of cementitious materials: volumetric relations and expansions, NISTIR 5390, Building and Fire Research Laboratory, Gaithersburg (MD), 1994.

[27] G.W. Scherer, Crystallization in pores, Cem. Concr. Res. 29 (1999) 1347-1358.

[28] W. Kunther, B. Lothenbach, K. Scrivener, Influence of bicarbonate ions on the deterioration of mortar bars under sulfate attack, Cem. Concr. Res. 44 (2013) 77-86.

[29] C. Yu, K. Scrivener, Mechanism of expansion of mortars immersed in sodium sulphate solution, Cem. Concr. Res. 43 (2013) 105-111.

[30] J.G.Wang, Sulfate attack on hardened cement paste, Cem. Concr. Res. 24 (1994) $735-42$.

[31] I. Oliveira, S.H.P. Cavalaro, A. Aguado, New kinetic model to quantify the internal sulfate attack in concrete, Cem. Concr. Res. 43 (2013) 95-104.

[32] S. Mindess, J. F. Young and D. Darwin, Concrete, Englewood. Cliffs. New Jersey. Prentice Hall, 2002.

[33] S.M. Abdelkader, Influencia de la composición de distintos hormigones en los mecanismos de transporte de iones agresivos procedentes de medios marinos, $\mathrm{PhD}$ thesis, Universidad Politécnica de Madrid, Madrid, 2010.

[34] R. Tixier and B. Mobasher, Modeling of damage in cementbased materials subjected to external sulfate attack. II: Comparison with experiments, J. Mater. Civ. Eng. 15 (2003) 314-322.

[35] W. Müllauer, R. E. Beddoe, D. Heinz, Sulfate attack expansion mechanisms, Cem. Concr. Res. 52 (2013) 208-215. 